\title{
Antibacterial activity of lavender essential oil and linalool combined with gentamicin on selected bacterial strains
}

\author{
MICHALINA ADASZYŃSKA-SKWIRZYŃSKA, DANUTA SZCZERBIŃSKA, SŁAWOMIR ZYCH* \\ Faculty of Biotechnology and Animal Husbandry, West Pomeranian University of Technology in Szczecin, \\ 29 Klemensa Janickiego Street, 71-270 Szczecin, Poland \\ *Labo-Wet Laboratory, Pyrzycka 9A Street, 70-892 Szczecin
}

\section{Adaszyńska-Skwirzyńska M., Szczerbińska D., Zych S. Antibacterial activity of lavender essential oil and linalool combined with gentamicin on selected bacterial strains}

\section{Summary}

The aim of this study was to verify the existence of the synergistic antibacterial effect of lavender essential oil and linalool individually combined with the antibacterial drug gentamicin. We investigated in vitro the effectiveness of the combinations of gentamicin and lavender essential oil and of gentamicin and linalool against the following strains: Staphylococus aureus ATCC 25923, Staphylococus aureus MRSA and Pseudomonas aeruginosa ATCC 9027. In order to determine the sensitivity of these microorganisms, we determined the minimum inhibitory concentration (MIC - Minimal Inhibitory Concentration). The study of the interaction of gentamicin with lavender oil and linalool was evaluated by the checkerboard method. Synergistic interaction between lavender essential oil and gentamicin and between linalool and gentamicin was observed against Staphylococcus aureus ATCC 25923 and Staphylococcus aureus MRSA. In particular, a very strong synergistic interaction was observed against Staphyloccocus aureus MRSA (lavender essential oil FIC index =0.14; linalool FIC index $=0.13$ ). In contrast, combinations of gentamicin and lavender essential oil and of gentamicin and linalool were less effective against Pseudomonas aeruginosa growth.

Keywords: lavender essential oil, gentamicin, antibacterial activity, synergism

The dynamic development of microbiology and medicine has advanced the study of pharmacology, veterinary medicine and the production of therapeutic agents. Although most medicines are produced by chemical synthesis, there has been a gradual increase of interest in the use of biologically active substances of plant origin in therapy and the prevention of a number of diseases $(13,19)$. Interest is also related to the fact that synthetic substances are characterised by many adverse effects and reduced effectiveness in the treatment of bacterial infections. Their reduced effectiveness can be attributed to the emergence of strains resistant to antibiotics, which is why it is necessary to search for substances with antimicrobial action (14, 19). With regard to the growing role of substances of plant origin in the treatment of infections of different aetiology, essential oils deserve special attention. This applies in particular to the essential oil of Lavandula angustifolia, which has antiseptic properties $(1,2,13$,
20). The antibacterial action of essential oils, including lavender essential oil, is proven, but their interaction with synthetic antibiotics is poorly known $(1,2,13,15)$.

Gentamicin is an aminoglycoside with strong bactericidal activity. Aminoglycosides disturb the biosynthesis of proteins, and their impact depends on concentration: high concentration boosts their biocidal action, while at lower concentrations bacteriostatic action can be observed (5). Aminoglycosides are used in treatments for humans, poultry, bees, cattle, pigs, sheep, goats, rabbits, camels and fish. They are effective as a remedy for sepsis and infectious diseases of the alimentary, respiratory and urinary tracts $(5,17)$. Gentamicin, which also belongs to this group, is used in the treatment of wound infections caused mainly by Staphylococcus aureus, resistant to $\beta$-lactams (MRSA - methicillin-resistant Staphylococcus aureus), and by Pseudomonas aeruginosa (multi-drug resistant). $S$. aureus and $P$. aeruginosa are versatile bacterial 
pathogens and common etiological agents in polymicrobial infections. Both $S$. aureus and $P$. aeruginosa exhibit intrinsic and acquired antibiotic resistance, making infections by these pathogens increasingly difficult to treat. Microbial communities containing both of these pathogens are shaped by interactions ranging from parasitic to mutualistic, with the net impact of these interactions in many cases resulting in enhanced virulence. Moreover, synergism between $S$. aureus and $P$. aeruginosa has been observed in multiple models of infection, including wounds and chronic lung infection. Since the 1980s no new aminoglycosides with high effectiveness and low toxicity have been developed. This has inhibited further work on new antibiotics in this group. Practical studies in the field focus on finding synergism between gentamicin and natural antimicrobial agents, such as essential oils. The synergism helps to reduce the medication dose to a level below its toxicity threshold (19). The aim of the study was to investigate the impact of lavender oil with gentamicin on selected bacterial strains.

\section{Material and methods}

Essential oil. A commercial lavender (Lavandula angustifolia) essential oil was used for the study. The essential oil manufacturer (Avicenna Oil, Wrocław, Poland) informs that the lavender oil was isolated by means of typical distillation with water vapor. The chromatographic analysis of the essential oil was performed using an Agilent $6890 \mathrm{~N}$ gas chromatograph with a $5973 \mathrm{~N}$ mass selective detector and a 7683 Series Injector. Samples for the analyses were prepared by dissolving essential oil $(0.02 \mathrm{~mL})$ in $1.0 \mathrm{~mL}$ of acetone (p.a.). The conditions of the chromatographic analyses were optimized to obtain good resolution of the analytes. An HP-5MSI column (30 m length, $0.25 \mathrm{~mm}$ I.D., $0.25 \mu \mathrm{m}$ film thickness) was used for the separation. The column temperature was programmed as follows: initial temperature $60^{\circ} \mathrm{C}$ (hold $3 \mathrm{~min}$ ), ramp rate $5^{\circ} \mathrm{C} / \mathrm{min}$, final temperature $300^{\circ} \mathrm{C}$ (hold $1 \mathrm{~min}$ ). The carrier gas was helium with a flow rate of $1.2 \mathrm{~mL} / \mathrm{min}$. The temperature of the injector was set at $250^{\circ} \mathrm{C}$, MS source: $230^{\circ} \mathrm{C}$, MS quad: $150^{\circ} \mathrm{C} .3 \mu \mathrm{L}$ of sample were injected via split (10:1) and injection of each sample was repeated three times to obtain averaged values. The ingredients of lavender essential oil from Avicenna Oil from Wrocław were described in previous publications $(2,3)$. The main components were linalool $35.17 \%$ and linalool acetate $46.25 \%(2-4)$. The chemical composition of the essential oil complied with the requirements of ISO 3515:2002 (www.iso.org/standard/36253. html) and the European Pharmacopoeia (9).

Microdilution Checkerboard Method. The anti-bacterial action of lavender essential oil (Avicenna Oil, Wrocław, Poland), its main ingredient - linalool (Merck, Darmstadt, Germany) - and gentamicin ( $256 \mathrm{mg} / \mathrm{mL}$; Oxoid Limited, Hampshire, Argentina) were determined by means of the Minimum Inhibitory Concentration (MIC) microdilution assay and verified according to CLSI recommendations (Clinical and Laboratory Standard Institute, 2002) Protocol M7-A6 (7). The dilutions were performed using DMSO (POCH, Gliwice, Poland). Two bacterial strains of the
American Type Culture Collection (S. aureus ATCC 25923, P. aeruginosa ATCC 9027) and one isolate from purulent skin lesions in a horse (S. aureus MRSA - bacteria from the culture collection of the Labo-Wet Laboratory, Szczecin, Poland) were used for the study.

The bacteria were inoculated by reductive culture into a Columbia Blood agar with 5\% of sheep blood (Oxoid) medium and incubated for $24 \mathrm{~h}$ at $37^{\circ} \mathrm{C}$. After 24 hours, 2-5 typical colonies of the working strain culture were collected and suspended in an isotonic, sterile $0.85 \% \mathrm{NaCl}$ solution. Next, optical density was measured with a density meter (measurement deviation of \pm 0.1 , in the $0.00 \div 3.00$ range according to McFarland's scale) causing turbidity of $0.5 \mathrm{MF}$, which corresponded to the average quantity of $1.5 \times 10^{8} \mathrm{CFU} / \mathrm{mL}$. All identifications were made on individually packed, sterile, divided, 96-wells polystyrene titration plates with flat bottoms. A series of two-fold dilutions of each antimicrobial agents was prepared in Mueller-Hinton broth (MHB, Oxoid) ranging from $0.002 \div 256 \mu \mathrm{g} / \mathrm{mL}$ for gentamicin, while for lavender oil and linalool, the concentration gradient was $0.008 \div 5 \% \mathrm{v} / \mathrm{v}$. At the next stage, $0.005 \mathrm{~mL}(=5 \mu \mathrm{L})$ of bacterial was added to each well (at the final concentration of $10^{5} \mathrm{CFU} / \mathrm{mL}$ in the well). In order to exclude an inhibitory effect of DMSO and possible contaminations, the positive and negative control assays were also performed.

The resulting checkerboard contains a combination of a concentration gradient of two antimicrobial agents, wherein wells that contain the highest concentration of each agent were located at opposite corners of a plate. Plates were sealed and incubated at $37 \pm 1{ }^{\circ} \mathrm{C}$ for 24 hours. At the end of the incubation, MIC was defined as the lowest concentration that did not result in any visible growth of the bacterial strains compared to their growth in the control wells (8). MIC determinations were realised in triplicate in three independent assays. Additionally, the result was recorded as the absorbance of individual plate wells using a $650 \mathrm{~nm}$ wavelength filter in a BioTek Elx800 microplate reader. MIC data of the essential oils and gentamicin were converted into Fractional Inhibitory Concentration (FIC) defined as the ratio of the concentration of the antimicrobial in an inhibitory concentration with a second compound to the concentration of the antimicrobial by itself (18). In the combination assays, the checkerboard procedure described by Rosato et al. (16) was followed to evaluate the synergistic action of the lavender essential oil or linalool with gentamicin. In our experimental protocol the substance combinations were analysed by calculating the FIC index (FICI) using the following formulas:

FIC of essential oil or linalool $=$ MIC value of combinated essential oil or linalool with gentamicin / MIC value of lavender essential oil or linalool alone

FIC of gentamicin $=$ MIC value of combinated gentamicin with essential oil or linalool / MIC value of gentamicin alone

FICI $=$ FIC value of essential oil or linalool + FIC value of gentamicin

Generally, the FICI value was interpreted as: synergistic when $\leq 0.5$; additive when $>0.5$ and $\leq 1$; noninteractive $(>1$ but $\leq 4)$ and antagonistic when $>4$ (18). 


\section{Results and discussion}

Susceptibility of the studied $S$. aureus ATCC 25923, $S$. aureus MRSA and P. aeruginosa ATCC9027 to lavender oil, linalool and gentamicin was identified by the microdilution method. Table 1 presents the MIC of lavender oil, linalool and gentamicin, which impede the growth of the studied microbes. The tested bacterial strains revealed sensitivity to lavender oil and linalool in concentrations from 0.25 to $2.0 \%(\mathrm{v} / \mathrm{v})$. $\mathrm{MIC}_{0}$ ( single sample), MIC (MIC of a single sample in the most effective combination) and FIC for lavender oil ranged from 0.25 to $1.25(\% \mathrm{v} / \mathrm{v})$, from 0.016 to $0.25(\% \mathrm{v} / \mathrm{v})$, and from 0.01 to $0.02(\% \mathrm{v} / \mathrm{v})$, respectively while the $\mathrm{MIC}_{0}, \mathrm{MIC}_{\mathrm{c}}$ and FIC for linalool ranged from 0.25 to $2.0(\% \mathrm{v} / \mathrm{v})$, from 0.008 to $0.75(\% \mathrm{v} / \mathrm{v})$ and from 0.03 to $0.75(\% \mathrm{v} / \mathrm{v})$ respectively. The same $\mathrm{MIC}_{0}$ of linalool for $S$. aureus ATCC 25923 and S. aureus MRSA was demonstrated by Carson and Riley (6), while a lower value of $0.125 \% \mathrm{v} / \mathrm{v}$ was obtained by Fisher and Philips (10). Horváth et al. (12) observed no impact of linalool on $S$. aureus MRSA. The studied bacterial strains varied in their sensitivity to gentamicin (Tab. 1). Susceptibility of $S$. aureus ATCC 25923 (MIC - 0.125 $\mu \mathrm{g} / \mathrm{mL}$ ) and P. aeruginosa ATCC 9027 (MIC $-2.0 \mu \mathrm{g} /$ $\mathrm{mL}$ ) was observed, while $S$. aureus MRSA (MIC-32.0 $\mu \mathrm{g} / \mathrm{mL}$ ) was identified as resistant.

The observed growing incidence of bacterial infections and resistance to the applied drugs drives the quest for new solutions to improve the effectiveness of treatments (14). Recent scientific publications have presented studies on biologically active substances of plant origin, such as essential oils $(1,2,13,19,20)$. The substances have a wide spectrum of antibacterial action but are less toxic than most chemical medications (19). From a clinical point of view, comparative studies, which identify the sensitivity of bacteria to essential oils in comparison with their sensitivity to standard antibacterial medicines, are highly valuable. Our studies on the impact of lavender oil and linalool in combination with gentamicin were based on the checkerboard method. The calculated FICI values were used to demonstrate the presence or lack of synergism, addition or presence of antagonism. Analyses of different gentamicin combinations with lavender oils revealed that they had diversified impact on microbes, beginning with the ones suggesting synergism $(S$. aureus ATCC 25923: lavender essential oil FICI - 0.192 and linalool FICI - 0.288; S. aureus MRSA: lavender essential oil FICI -0.138 and linalool FICI -0.127 ), through to an additive effect ( $P$. aeruginosa ATCC 9027: lavender essential oil FICI - 0.700), and lack of interaction (P. aeruginosa ATCC 9027: linalool FICI - 1.25). One should note that a synergism of gentamicin and lavender oil, and linalool impact towards the resistant strain ( $S$. aureus MRSA) was achieved. In none of the cases was antagonistic action observed. The experiments carried out by other authors showed synergism of other essential oils with gentamicin, towards Gram positive and Gram negative bacteria $(11,15,16,20)$, as confirmed by proprietary studies on lavender oil, whose combinations with gentamicin have not been investigated before. The growing resistance of many bacterial strains to medicines that has been observed in the last decades have prompted contemporary medicine and veterinary medicine to follow combined treatments. Only a few publications have reported higher antibacterial activity of antibiotics used together with essential oils in in vivo tests (11). Ismail et al. (11) demonstrated the effectiveness of treating rats with black pepper (Piper nigrum) essential oil in combination with antibacterial medicines, e.g. gentamicin. The results obtained by Ismail et al. (11) suggest that black

Tab. 1. Lavender essential oil and linalool in combination with gentamicin - Fractional Inhibitory Concentration (FIC) and FIC Indices (FICI)

\begin{tabular}{|c|c|c|c|c|c|c|c|c|c|}
\hline & \multicolumn{4}{|c|}{ Lavender Essential Oil } & \multicolumn{4}{|c|}{ Linalool } & \multirow{2}{*}{ Type of interaction } \\
\hline & $\mathrm{MIC}_{0}$ & $\mathrm{MIC}_{\mathrm{c}}$ & FIC & FICI & $\mathrm{MIC}_{0}$ & $\mathrm{MIC}_{\mathrm{c}}$ & FIC & FICI & \\
\hline \multicolumn{10}{|c|}{ Staphylococcus aureus ATCC 25923} \\
\hline Essential oil/linalool (\% v/v) & 0.250 & 0.016 & 0.064 & \multirow{2}{*}{0.192} & 0.250 & 0.008 & 0.032 & \multirow{2}{*}{0.288} & \multirow{2}{*}{ synergistic } \\
\hline Gentamicin $(\mu \mathrm{g} / \mathrm{mL})$ & 0.125 & 0.016 & 0.128 & & 0.125 & 0.032 & 0.256 & & \\
\hline \multicolumn{10}{|c|}{ Staphylococcus aureus MRSA } \\
\hline Essential oil/linalool (\% v/v) & 1.25 & 0.016 & 0.013 & \multirow{2}{*}{0.138} & 0.250 & 0.016 & 0.064 & \multirow{2}{*}{0.127} & \multirow{2}{*}{ synergistic } \\
\hline Gentamicin $(\mu \mathrm{g} / \mathrm{mL})$ & 32.0 & 4.0 & 0.125 & & 16.0 & 1.0 & 0.063 & & \\
\hline \multicolumn{10}{|c|}{ Pseudomonas aeruginosa ATCC 9027} \\
\hline Essential oil/linalool (\% v/v) & 0.625 & 0.250 & 0.200 & \multirow{2}{*}{0.700} & 2.0 & 1.5 & 0.750 & \multirow{2}{*}{1.250} & \multirow{2}{*}{ additive or no interaction } \\
\hline Gentamicin $(\mu \mathrm{g} / \mathrm{mL})$ & 2.0 & 1.0 & 0.5 & & 2.0 & 1.0 & 0.5 & & \\
\hline
\end{tabular}

Explanations: MIC - Minimal Inhibitory Concentration; FIC - Fractional Inhibitory Concentration; FICI - Fractional Inhibitory Concentration Index; $\mathrm{MIC}_{0}=\mathrm{MIC}$ of an individual sample alone; $\mathrm{MIC}_{\mathrm{c}}=\mathrm{MIC}$ of an individual sample of the most effective combination; FIC of oil or linalool $=$ MIC of oil or linalool in combination with gentamicin/ $\mathrm{MIC}$ of oil or linalool alone; FIC of gentamicin $=\mathrm{MIC}$ of gentamicin in combination with oil or linalool/MIC of gentamicin alone; FIC index = FIC of oil or linalool + FIC of gentamicin; FICI $\leq 0.5$, synergistic; FICI $>0.5-1.0$, additive; FICI $>1.0-4.0$, no interaction; FICI $>4.0$, antagonistic (19) 
pepper essential oil has a strong synergic action when combined with gentamicin and ciprofloxacin, while not having a toxic impact on renal and hepatic functions when rats are fed with a value ten times higher than MIC.

The following three types of interactions were discovered among the studied combinations of gentamicin with lavender oil and linalool: synergism, additivity and neutrality. In combinations towards $S$. aureus, only synergistic impacts on the resistant strain were observed. The combination of gentamicin and linalool towards $S$. aureus MRSA turned out to be the most effective combination (with the strongest bactericidal action), while the combination of gentamicin with lavender oil towards $P$. aeruginosa ATCC 9027 was the least effective. Gentamicin MIC reduction against $S$. aureus MRSA through pharmacological treatment is very difficult nowadays. These important results may help the formulation of new topical agents for the cure of infections caused by the Gram positive bacteria. In light of the presented results, studies on combinations of antibiotics and essential oils and their ingredients should be continued with regard to their potential antibacterial effectiveness. Finally, we suggest that this study could provide a platform for researchers to perform further experimental procedures in order to shed more light on the mechanism of action of the synergism and plan clinical trials to confirm these in vitro results.

\section{References}

1.Adaszyńska M., Swarcewicz M., Dzięciol M., Dobrowolska A.: Comparison of chemical composition and antibacterial activity of lavender varieties from Poland. Nat. Prod. Res. 2013, 27, 1497-1501.

2. Adaszyńska-Skwirzyńska M., Szczerbińska D.: The antimicrobial activity of lavender essential oil (Lavandula angustifolia) and its influence on the production performance of broiler chickens. J. Anim. Physiol. Anim. Nutr. 2018, 102, 1020-1025.

3. Adaszyńska-Skwirzyńska M., Szczerbińska D.: The effect of lavender (Lavandula angustifolia) essential oil as a drinking water supplement on the production performance, blood biochemical parameters, and ileal microflora in broiler chickens. Poult. Sci. 2019, 98, 358-365.
4. Babushok V. I., Linstrom P. J., Zenkevich I. G.: Retencion indices for frequently reported compounds of plant essential oils. J. Phys. Chem. Ref. Data 2011 40, 2-47.

5. Backer B., Cooper M. A.: Aminoglycoside Antibiotics in the $21^{\text {st }}$ Century. ASC Chem. Biol. 2013, 8, 105-115.

6. Carson C. F., Riley T. V.: Antimicrobial activity of tea tree oil. Rural Ind. Res. RIRDC Pub. 1998, 9, 1-51

7. Clinical Laboratory Standards Institute: Methods for dilution antimicrobial susceptibility tests for bacteria that grow aerobically, approved standard: M7-A6, 2002, vol. 23-n ${ }^{\circ}$.

8. European Committee for Antimicrobial Susceptibility Testing (EUCAST) Terminology relating to methods for the determination of susceptibility of bacteria to antimicrobial agents, in Eucast definitive document E.Def. 1.2., European Society of Clinical Microbiology and Infectious Diseases (ESCMID). Basel (SW), 2002, 6, 503-508

9. European Pharmacopeia 8.0-8.8. (Eur Ph). 2014.

10. Fisher K., Phillips C. A.: The effect of lemon, orange and bergamot essential oils and their components on the survival of Campylobacter jejuni, Escherichia coli O157, Listeria monocytogenes, Bacillus cereus and Staphylococcus aureus in vitro and in food systems. J. Appl. Microbiol. 2006, 101, 1232-1240.

11. Ismail M., Kemegne G. A., Njayou F. N., Penlap V., Mbacham W. F., Kamdem S. L. S.: Chemical composition, antibiotic promotion and in vivo toxicity of Piper nigrum and Syzygium aromaticum essential oil. Afri. J. Biochem. Res. 2017, 11, 58-71.

12. Horváth G., Jambor N., Vegh A., Böszörményi A., Lemberkovics E., Héthelyi E., Kovácsc K., Kocsis B.: Antimicrobial activity of essential oils: the possibilities of TLC-bioautography. Flavour Frag. J. 2010, 25, 178-182.

13. Hossain S., Heo H., De Silva B. C. J., Wimalasena S. H. M. P., Pathriana H. N. K. S., Heo G. J.: Antibacterial activity of essential oil from lavender (Lavandula angustifolia) against pet turtle-borne pathogenic bacteria. Lab. Anim. Res. 2017, 33, 195-201.

14. Munita J. M., Ariasa C. A.: Mechanisms of antibiotic resistance. Microbiol. Spectrum 2016, 4, 1-37.

15. Moussaoui F., Alaoui F.: Evaluation of antibacterial activity and synergistic effect between antibiotic and the essential oils of some medicinal plants. Asian Pac. J. Trop. Biomed. 2016, 6, 32-37.

16. Rosato A., Piarulli M., Corbo F., Muraglia M., Carone A., Vitali M. E., Vitali C.: In vitro synergistic action of certain combinations of gentamicin and essential oils. Curr. Med. Chem. 2010, 17, 3289-3295

17. Vaarten J.: Clinical impact of antimicrobioal resistance in animals. Rev. Sci. Tech. Int Office Epizoot. 2012, 31, 221-230.

18. Van Vuuren S., Viljoen A.: Plant-based antimicrobial studies. Methods and approaches to study the interaction between natural products. Planta Med. 2011, 77, 1168-1182.

19. Veras H. N. H., Rodrigues F. F. G., Botelho M. A., Menezes I. R. A., Coutinho H. D. M., Costa J. G. M.: Enhancement of aminoglycosides and $\beta$-lactams antibiotic activity by essential oil of Lippia sidoides Cham. and the thymol. Arab. J. Chem. 2017, 10, 2790-2795.

20. Yap P. S., Yiap B. Ch., Ping H. C., Lim S. H. E.: Essential oils, a new horizon in combating bacterial antibiotic resistance. Open Microbiol. J. 2014, 8, 1-6.

Corresponding author: Michalina Adaszyńska-Skwirzyńska PhD, 29 Klemensa Janickiego Street, 71-270 Szczecin, Poland; e-mail: madaszynska@zut.edu.pl 